\title{
CHANNEL MODELING USING THE PARABOLIC EQUATION FOR RF COMMUNICATIONS
}

\author{
V. Gadwal \\ A. Barrios \\ Space and Naval Warfare Systems Center Pacific (SSC Pacific) \\ Atmospheric Propagation Branch, Code 55480 \\ 49170 Propagation Path \\ San Diego, California
}

\begin{abstract}
A method that uses the parabolic equation (PE) model to characterize the transmission channel for wideband waveforms is discussed. The results presented use the Advanced Propagation Model (APM) developed by the Atmospheric Propagation branch at the SSC Pacific which utilizes the split-step Fourier algorithm to solve the PE wave equation for a continuous wave $(C W)$ source. To determine the channel response for a given geometry and environment, an approach used to determine the proper frequency sampling to resolve the multipath components will be discussed. The channel impulse response is computed for an ideal case having a direct and reflected path with a known solution, as well as for examples using ducting, terrain, and a surface-based duct measured off the coast of southern California. An example is provided to demonstrate how this method can be used to characterize the time spreading of a waveform and how it can be used to assess the impact of channel-induced intersymbol interference (ISI).
\end{abstract}

\section{INTRODUCTION}

The APM has been developed by the Atmospheric Propagation branch at the SSC Pacific to provide the Navy with a tool for assessing the effects of atmospheric conditions on radiowaves. This model has proven to perform very well for predicting field strength from VHF to Ka-band for both over-water and over-terrain propagation paths in the presence of range-dependent refractive conditions for surface-to-surface, surface-to-air, and air-to-air geometries. The APM is used not only by the U.S. operational communities, but also by many civilian and homeland defense agencies. Although this model provides a powerful tool for radar performance assessment, it does not provide the same capability for assessing the performance of modern communication systems. With heavy reliance on wireless communications, an accurate characterization of the propagation channel is vital. The current method of determining propagation loss for single frequency sources is insufficient for RF digital communications applications. Proper characterization of the transmission channel for frequency-hopper and other wideband waveforms is required for determining the quality of the communication link.

The APM uses the split step parabolic equation (PE) model to determine the complex field (amplitude and phase) of a $\mathrm{CW}$ source. The PE model provides an approximation to the scalar wave equation modeling electromagnetic waves propagating at small angles from the transmitter in range by assuming that the field varies slowly in this direction. The advantage of this model is that it gives a computationally efficient full wave solution of the field in the presence of terrain and range dependent environments. The channel transfer function for a wideband channel is computed by dividing the bandwidth of interest into multiple frequency bins with appropriate spacing and computing the complex field at each bin. The impulse response is then computed by taking the inverse discrete Fourier transform of the transfer function, where the bandwidth used determines the time resolution of the impulse response and the frequency spacing determines its time window.

This paper will describe in detail the method used to implement the wideband channel model, and show examples that were run to gain a further understanding of the model. The impulse response is computed over water for two cases. The first uses a standard environment where the multipath arrivals at the receiver consist of direct and reflected ray paths, the second is for an environment with a 300 meter surface-based duct present. For these cases, the impulse response can be verified by using a geometric ray trace to compute the arrival times of the multipath returns. Ray tracing is not applicable when the terrain is included. Two examples will be shown where the impulse response is computed for terrain cases, one using a wedge terrain and a standard environment, and the other using a real environment and terrain. The multipath returns at the receiver may give rise to intersymbol interference (ISI) in a communication system. Time domain symbols can be simulated based on pulse shaping, modulation type, and 
the data rate used by the system. These symbols can be convolved with the impulse response to simulate the effect of the channel on the ISI in the system.

\section{WIDEBAND IMPLEMENTATION}

The wide-angle PE algorithm is employed to evaluate the amplitude and phase of a CW source for a given geometry. The PE method is derived from the two dimensional scalar wave equation in the $x$ (range) and $z$ (height) directions. The PE model works based on the assumption that the field is slowly varying in the $x$ direction. This allows the removal of the rapidly varying phase fluctuations from the field in the $\mathrm{x}$ direction. The complex field is computed by taking spatial Fourier transforms in the $z$ direction and then "marching" it forward in the $x$ direction, making this algorithm an efficient method to compute a full wave solution of the field. As implemented in the APM, the maximum $\mathrm{PE}$ angle; i.e., the maximum propagation angle the PE algorithm can accurately model in the field computation, does not exceed twenty degrees. The wideangle propagator is written as

$$
u(x+\Delta x)=e^{i k_{0} \Delta x 10^{-6} M(z)} F^{-1}\left\{e^{i \Delta x \sqrt{k_{0}^{2}-p^{2}}-k_{0}} F\{u(x, z)\}\right\}
$$

where $k_{0}$ is the free space wavenumber, $\Delta x$ is the incremental range step over which the solution is propagated, $M(z)$ is the modified refractivity profile, and $p$ is the spatial wavenumber written as $k_{0} \sin \theta$, where $\theta$ is the propagation angle. Since the algorithm models twodimensional, forward propagation, effects such as lateral diffraction and back-scattering have been neglected.

For computational efficiency fast Fourier transforms (FFTs) are used by the PE algorithm to go from the spatial, $z$, domain to the spatial frequency, $p$, domain. In doing so the height step, $\Delta z$, is determined by the Nyquist criteria as

$$
\Delta z=\frac{c}{2 f \sin \theta_{\max }}
$$

where $\theta_{\max }$ denotes the PE angle selected each time the algorithm is run, $c$ is the speed of light, and $f$ is the source frequency. The algorithm is run multiple times to compute the transfer function of the channel by obtaining the amplitude and phase of the frequencies selected over the bandwidth of interest. Each time the algorithm is run $\Delta z$ can be kept constant to ensure that the complex field is evaluated at the exact receiver height. By fixing $\Delta z$, as $f$ increases, $\theta_{\max }$ decreases. There are two considerations to keep in mind when selecting $\theta_{\max }$. First, $\theta_{\max }$ needs to be large enough so that the field consists of every multipath arrival present at the receiver. Second $\theta_{\max }$ must not be large enough to violate the assumptions made by the PE model.

Ray tracing is utilized to determine $\theta_{\max }$ for a specific system geometry. By employing a simple geometric ray tracing tool, the number of multipath arrivals, the arrival times of each ray, as well as the elevation angles from the transmitter to which the rays are traced to the receiver can be determined. By computing the highest magnitude of the elevation angles, the minimum value of $\theta_{\max }$ that is needed to run the PE algorithm across the frequency band is determined. The fact that for a given range the transfer function for multiple receiver height locations can be computed using the same number of PE runs is exploited. When computing the transfer function at multiple receiver heights, the minimum value of $\theta_{\max }$ should take into account each ray traced to every receiver location. The minimum value of $\theta_{\max }$ corresponds to the $\mathrm{PE}$ angle chosen for the stopping frequency in the bandwidth of interest. The value of the PE angle at the stopping frequency determines the PE angle at starting frequency, which must not be large enough to violate the assumptions made by the model. These angles limit the amount of bandwidth for which the channel can be modeled using this approach.

The ray tracing utility can only be used for cases in which terrain is not included. When terrain is present the PE angle needed to encompass all of the multipath arrivals should be kept large to ensure that all of the refractive effects are taken into account. The approach used to determine the PE angles for a given bandwidth is to calculate the angles to be as large as possible without violating the assumptions of the model.

The transfer function is computed by running the PE algorithm over the selected frequency band and frequency spacing. The frequency parameters are determined by the time domain parameters. Specifically, the time resolution of the impulse response is given by the inverse of the bandwidth, and the time window over which the impulse response is computed sets the inverse of the frequency spacing. The time resolution can be chosen to be small enough to resolve the time differences between all of the multipath arrivals, while the time window must be chosen to be long enough to contain every multipath arrival, otherwise aliasing will occur. The time domain parameters can be estimated using ray tracing. Using the wide-angle propagator, the complex field can be computed for a $\mathrm{CW}$ source with an angular frequency $\omega$, where we substitute $k=\omega / c$, for a receiver at range $x$ and height $z$, and denote the field by $u(x, z, \omega)$. The transfer function at this receiver location is written 


$$
H(\omega, x, z)=u(x, z, \omega)
$$

The impulse response at baseband is obtained using the inverse discrete Fourier transform as follows

$$
h\left(t_{m}\right)=N d f \sum_{n=0}^{N-1} H\left(\omega_{n}, x, z\right) e^{\frac{i 2 \pi m n}{N}}
$$

where $N$ is the number of frequencies over which the transfer function is computed and $t_{m}=x / c+m d t$ for $m=0,1, \ldots, N-1$.

\section{IMPULSE RESPONSE RESULTS}

The impulse response results are presented for four cases. All cases are computed for an omni-directional antenna and for horizontal polarization. First, a simple case is considered for energy propagating over water in a standard environment. This case has a known solution for the arrival times of the direct and reflected rays, offering a way to verify the results. Next the impulse response computation is verified using ray tracing for energy propagating over water in an environment with a $300 \mathrm{~m}$ surface-based duct. In this environment there are multiple arrivals present depending on the geometry used. After verifying the impulse response computation, terrain is included. A simple wedge is used to evaluate the effects of terrain. Finally, the effects that can be present in a real environment are demonstrated using a real terrain profile and using radiosonde data taken off the coast of Southern California. Channel illustrations, depicting the propagation medium effects reflected by the ray paths, are shown in Figure 1.

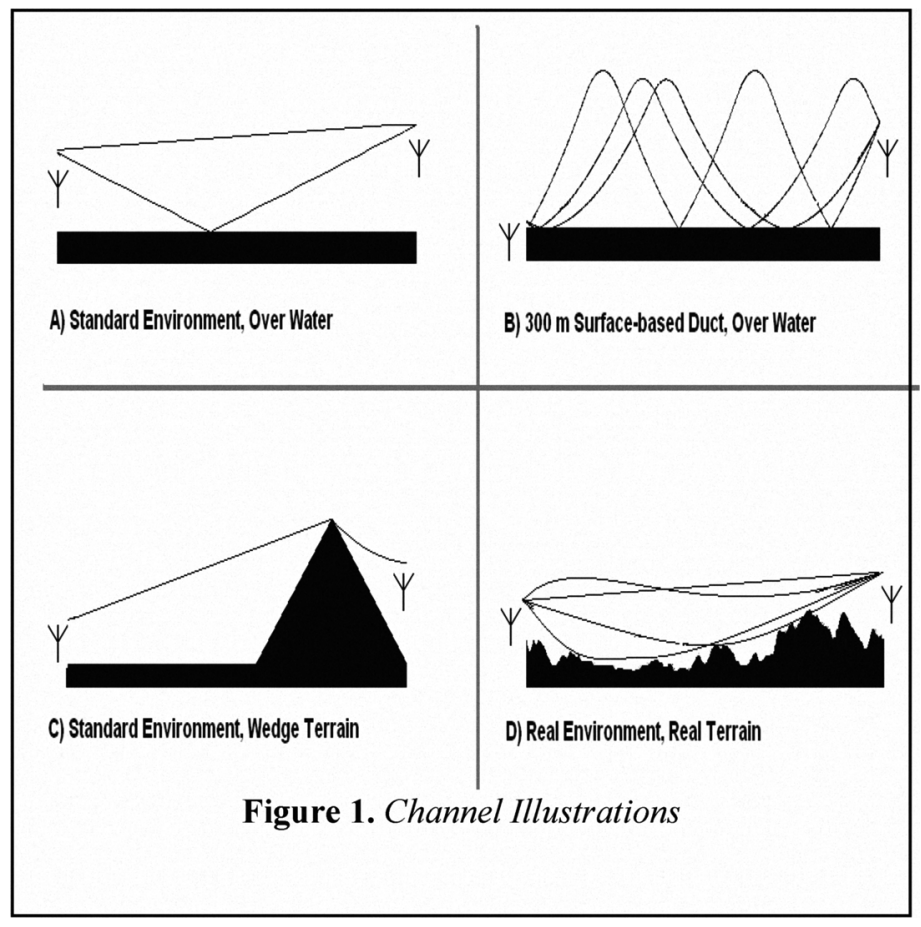

\section{A. Standard Environment, Over Water}

The impulse response is computed for a transmitter height of $150 \mathrm{~m}$, a receiver range of $5 \mathrm{~km}$, and three receiver heights at $50 \mathrm{~m}, 200 \mathrm{~m}$, and $500 \mathrm{~m}$. The impulse response was computed for a receiver height of $200 \mathrm{~m}$ in [3] from which the results can be compared. The result is also verified by noting that the arrival time of the direct ray at the $500 \mathrm{~m}$ receiver should be the same as the arrival time of the reflected ray at the $200 \mathrm{~m}$ receiver. The time difference between the direct and reflected arrivals is approximately $10 \mathrm{~ns}$ at the $50 \mathrm{~m}$ height, $40 \mathrm{~ns}$ at $200 \mathrm{~m}$, and $100 \mathrm{~ns}$ at $500 \mathrm{~m}$. The time difference between the first and last arrival to all of the receiver heights is approximately 140ns. Based on these values a time step of $7.8 \mathrm{~ns}$ and an overall time window of lus have been chosen. These values set the bandwidth to $128 \mathrm{MHz}$ and the frequency spacing to $1 \mathrm{MHz}$. The center frequency is chosen as $200 \mathrm{MHz}$. These parameters are chosen not for a specific system, but to attempt to resolve the multipath arrivals.

Figure 2 shows the results obtained after using an inverse discrete Fourier transform. The values shown in the parenthesis are the actual delay differences determined using ray tracing and the values outside the parentheses are the model estimates. Since the time step chosen is $7.8 \mathrm{~ns}$, the estimated values are within the margin of error.

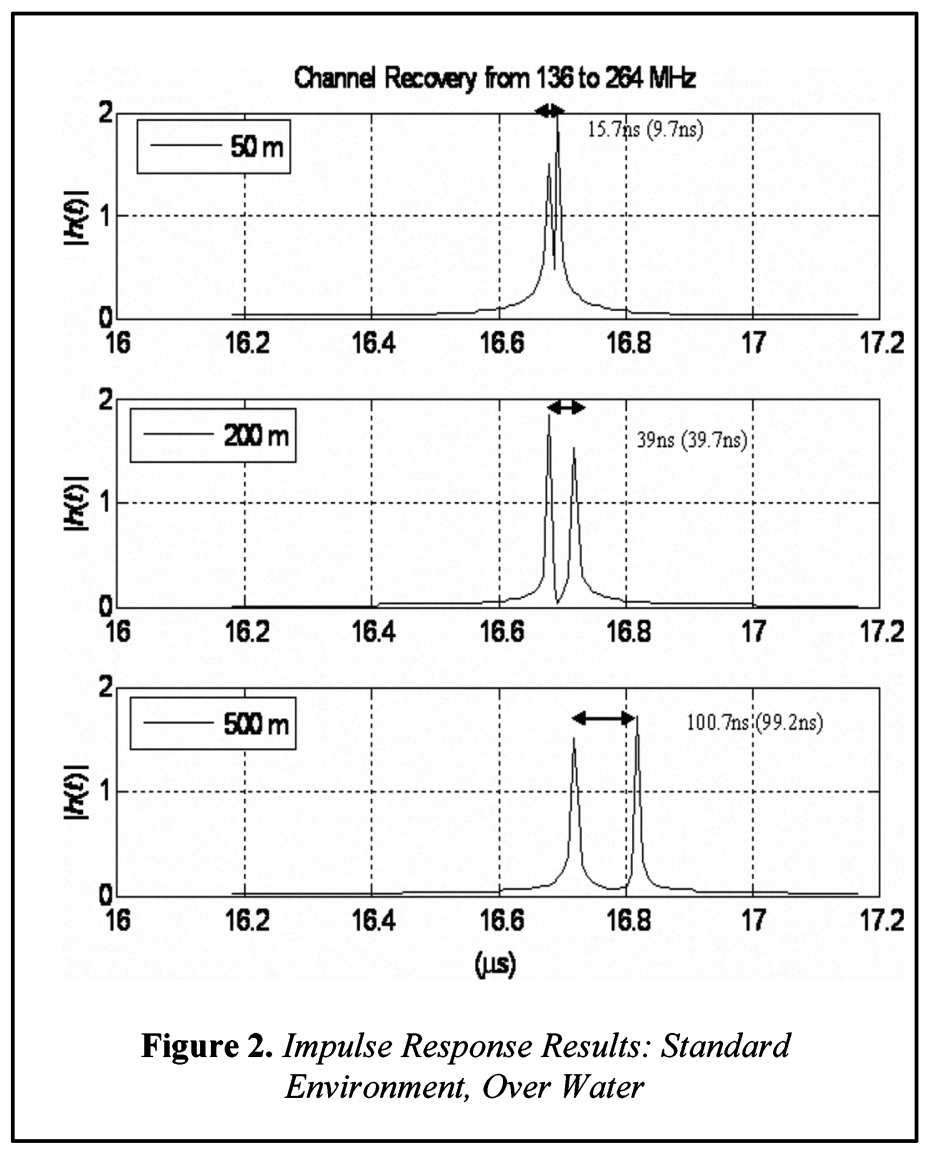




\section{B. 300 m Surface-based Duct, Over Water}

The impulse response is computed for a transmitter height of $25 \mathrm{~m}$, a receiver range of $200 \mathrm{~km}$, and three receiver heights at $50 \mathrm{~m}, 200 \mathrm{~m}$, and $500 \mathrm{~m}$. Using ray tracing, two arrivals are predicted at the $50 \mathrm{~m}$ receiver height. The time difference between these arrivals is 2 ns. There are three arrivals predicted at the $200 \mathrm{~m}$ receiver height. The predicted delay difference between the first and second arrivals is $3.7 \mathrm{~ns}$ and between the second and third is $8.3 \mathrm{~ns}$. The receiver at the $500 \mathrm{~m}$ height is outside of the duct and no arrivals are predicted. The time difference between the first and last arrival of all of the receiver heights is approximately $12 \mathrm{~ns}$. Based on these values, a time step of $1 \mathrm{~ns}$ and an overall time window of $128 \mathrm{~ns}$ have been chosen. These values set the bandwidth to $1000 \mathrm{MHz}$ and the frequency spacing to $7.8 \mathrm{MHz}$. The center frequency is set to $1500 \mathrm{MHz}$.

Figure 3 shows the impulse responses obtained using the parameters described above. The time step is $1 \mathrm{~ns}$ and is not enough to resolve the two arrivals at the $50 \mathrm{~m}$ receiver height. The values of the actual differences and the estimates at $200 \mathrm{~m}$ are in agreement since the difference between the values is within $1 \mathrm{~ns}$. The $500 \mathrm{~m}$ receiver result shows a weak arrival which is expected.

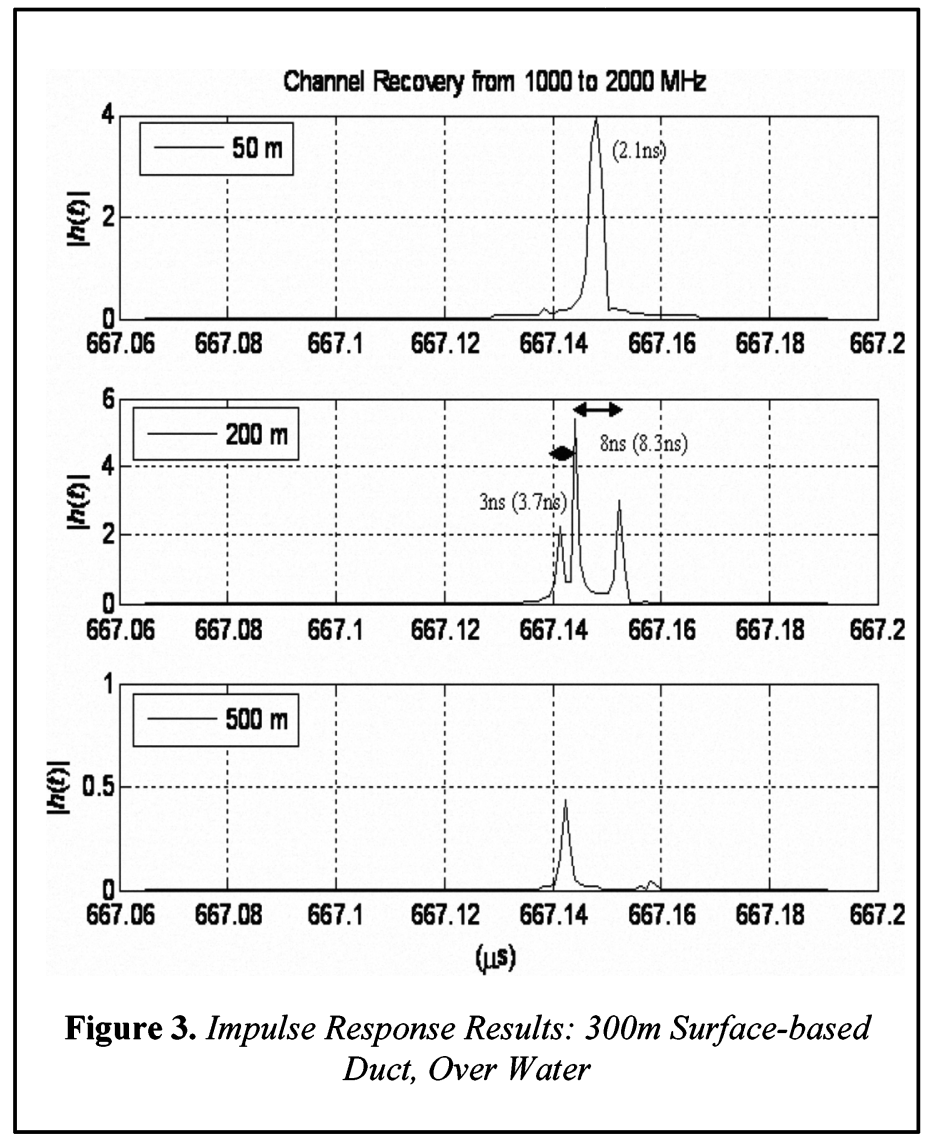

\section{Standard Environment, Wedge Terrain}

In the previous two cases, a geometric ray trace is used to determine the PE angle needed to account for all of the multipath arrivals. The determination of the bandwidth and the frequency spacing was based on the arrival times determined by the ray trace. This capability is not available when terrain is included. A higher PE angle will be needed to account for the interaction of the field with the terrain. The approach used to determine the PE angles is to keep them as high as possible over the bandwidth used without violating the assumptions made by the model. As a starting point, the geometric ray trace can determine the bandwidth and frequency spacing to use when excluding the terrain. These values are increased in order to define the same parameters when terrain is included.

The impulse response is computed for a transmitter height of $25 \mathrm{~m}$, a receiver range of $60 \mathrm{~km}$, and four receiver heights at $50 \mathrm{~m}, 200 \mathrm{~m}, 500 \mathrm{~m}$, and $1001 \mathrm{~m}$. The wedge extends from $40 \mathrm{~km}$ to $60 \mathrm{~km}$ in range and its peak is at $200 \mathrm{~m}$. The bandwidth chosen is $1500 \mathrm{MHz}$ and the frequency spacing is $5.9 \mathrm{MHz}$ giving a time step of $.67 \mathrm{~ns}$ and a time window of $171 \mathrm{~ns}$. The center frequency is set to $1000 \mathrm{MHz}$.

Figure 4 shows the impulse responses at the four receiver heights. To understand these results, a comparison is done to the results (not shown) obtained for the same geometry

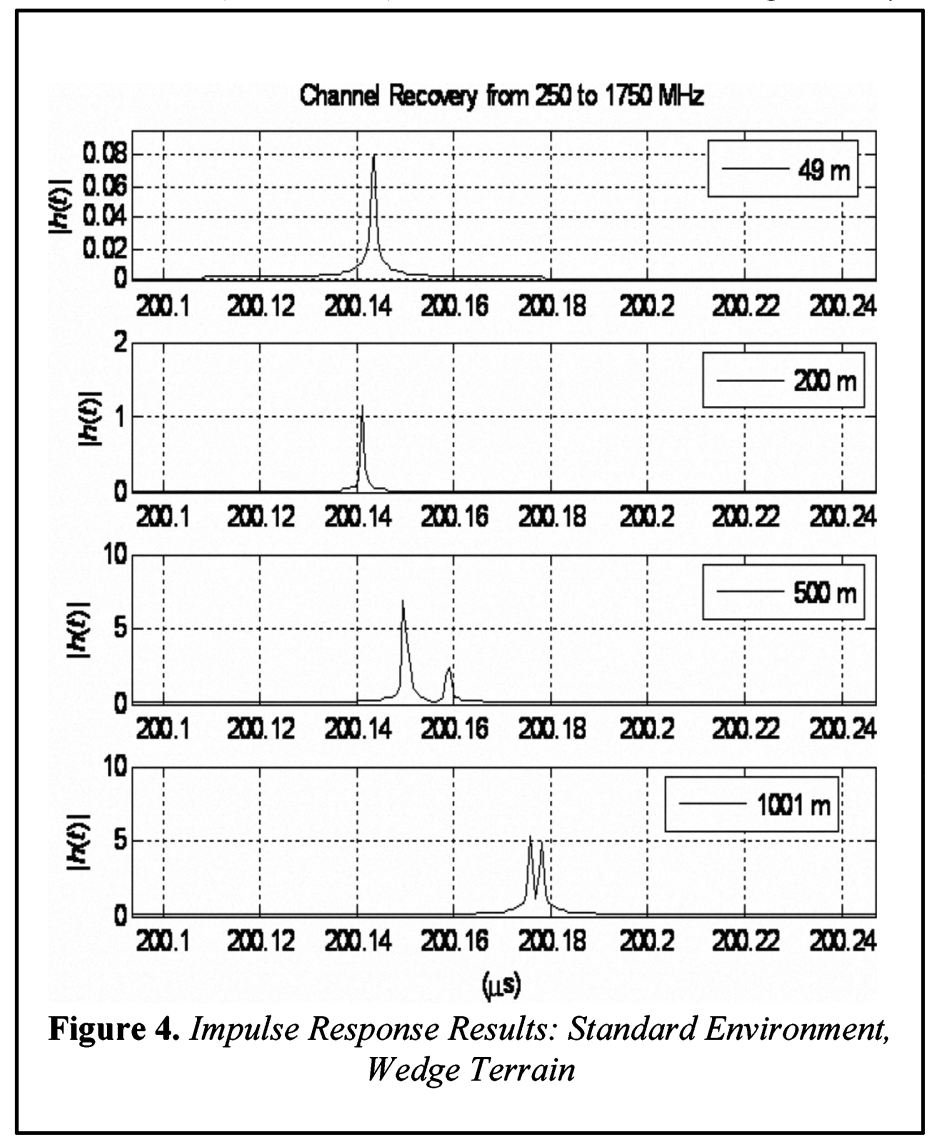


and environment, but taken instead over water. With terrain, the $50 \mathrm{~m}$ receiver does not have line of sight to the transmitter. The result is that the arrival at this receiver is delayed further, is weaker, and the pulse is wider than the arrival excluding terrain. The arrival at the $200 \mathrm{~m}$ receiver is also weaker than the over water case. At $500 \mathrm{~m}$, the diffraction due to the wedge peak should cause a greater number of multipath arrivals than when terrain is excluded. The second peak shown in Figure 4 for this receiver is not present when terrain is excluded, so this extra arrival that has been resolved is due to the diffraction effects. The results are practically equivalent at the $1001 \mathrm{~m}$ receiver because the receiver is high enough that the wedge does not significantly affect the multipath returns.

\section{Real Atmospheric Environment, Real Terrain}

Finally to demonstrate the effects that can be present in real atmospheric environments, a measured $400 \mathrm{~m}$ surfacebased duct taken from a radiosonde off the coast of Southern California is used along with a real terrain profile (shown in Fig. 1D). The transmitter height is $25 \mathrm{~m}$, the receiver range is $80 \mathrm{~km}$ and the receiver heights are $100 \mathrm{~m}$, $200 \mathrm{~m}, 500 \mathrm{~m}$, and $1001 \mathrm{~m}$ above ground level. Figure 5 shows the impulse response for the four receiver heights. This result demonstrates the need to consider these channel effects when evaluating the performance of high-bit-rate systems.

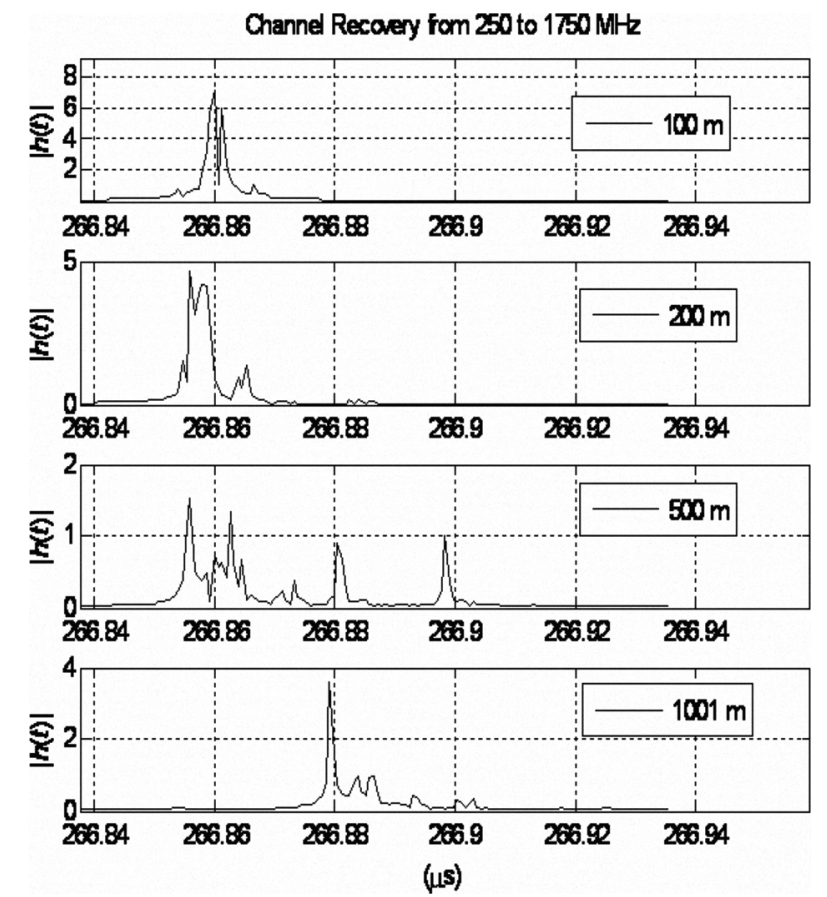

Figure 5. Impulse Response Results: Real Atmospheric Environment, Real Terrain

\section{WAVEFORM APPLICATION}

In this section a channel impulse response is applied to a transmitted waveform to show how this work can be used for RF system analysis. The multipath arrivals caused by the channel can create intersymbol interference in a system. ISI occurs when a transmitted symbol spreads into successive symbols. This will degrade the performance of a system by creating distortion in the waveform, decreasing the receiver's ability to correctly detect the signal. ISI is caused when a channel exhibits frequency selective fading in which the multipath arrivals are spread across a duration longer than the symbol time.

The ISI can be measured by using an eye diagram. An eye diagram is constructed by overlapping the received symbols across a time window of a fixed number of symbols. The symbols create an eye pattern where the time to sample the signal is towards the center of the eye. As the ISI increases, the eye pattern will become narrower due to the increased amplitude jitter at the sampling time which decreases the noise margin and increases the bit errors in the system.

An eye diagram can be simulated to apply the channel model to a digital system in order to understand the channel's effect on its performance. For this example, symbols are constructed using binary phase shift keying (BPSK) modulation, raised cosine filtering, and a data rate of $30 \mathrm{Mbps}$. The transmitted symbols are convolved with the channel impulse response. For this example, the impulse response at the $1001 \mathrm{~m}$ receiver from the real atmospheric environment, real terrain case is used (Section D). Figure 6 shows the result. The channel's effect on system performance can be qualitatively understood using this method. This example shows that the ISI caused by

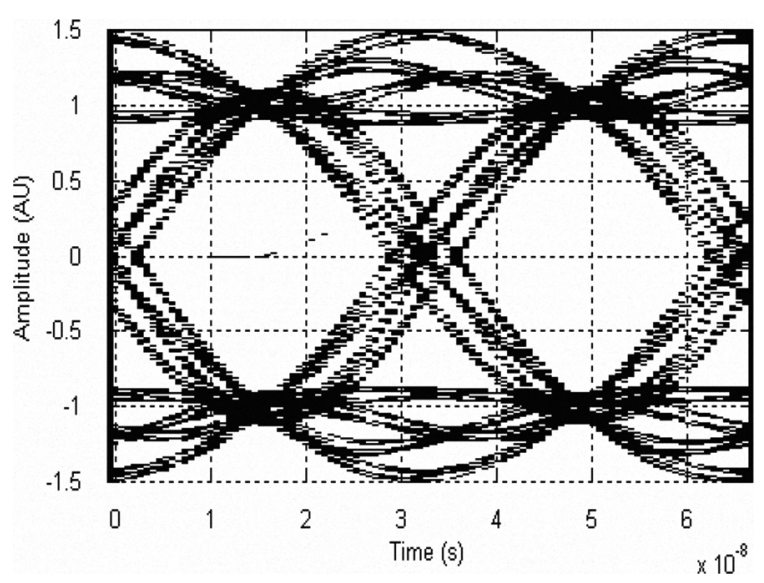

Figure 6. Simulated Eye Diagram 
the channel for this particular case should not have a significant impact on system performance.

A desirable performance metric is the system's bit error rate (BER). This can be achieved by using the eye diagram to determine the noise due to ISI and including this loss into a system link budget. A more accurate way of determining the BER for a given system would be to simulate the blocks of the system and compute the bit errors. The modulation, coding schemes, detector, and the noise in a system should be taken into account.

\section{CONCLUSIONS}

Evaluating the performance claims of wideband communication systems will require a credible RF channel model that arises from the needs of the U. S. Navy, as well as the other military services. US Navy shipboard systems will have to contend against surface and evaporation ducts which create complex propagation environments that can significantly impact system performance. The research done here leverages off an enormous investment made by ONR in the APM. The APM computes the 2D propagation of a narrowband source and determines the effects due to real terrain and range-dependent atmospheric conditions. The need to extend this model to perform wideband channel modeling has been addressed.

This paper has presented a discussion of the issues that arise in the implementation of the wideband channel model and has presented an approach to understanding the channel's impact on system performance. The channel impulse response has been computed for cases in which the results could be verified using a geometric ray trace. After verifying that the model is performing as expected for cases run over water, the channel response was computed for terrain cases. The real atmospheric environment, real terrain case demonstrated the importance of assessing these channel effects since the multipath distortion could have a significant impact on the performance of high-bit-rate systems. Finally, an eye diagram has been used to demonstrate how to qualitatively measure the ISI in a given system. Exploring the model further to understand the limitations on the amount of bandwidth that can be used, as well as developing an algorithm to run the model for all environment and terrain cases, and understanding the channel's effect on the BER of a system will all be explored further in future work.

\section{REFERENCES}

[1] Barrios, A. E., Considerations in the Development of the Advanced Propagation Model (APM) for the
U.S. Navy Applications, IEEE Proceedings of the International Radar Conference, 2003.

[2] Levy, M., Parabolic Equation Methods for Electromagnetic Wave Propagation, The Institute of Electrical Engineers, 2000.

[3] Palud, M. Le, Terrestrial Wireless Channel Modeling, Communications for Network-Centric Operations: Creating the Information Force, MILCOM, 2001.

[4] Craig, K.H., M. Levy, Field Strength Forecasting with the Parabolic Equation: Wideband Applications, International Conference on Antennas and Propagation, 1989.

[5] Gingras, D.F., P. Gerstoft, The Effect of Propagation on Wideband DS-CDMA Systems in the Suburban Environment, The First IEEE Signal Processing Workshop on Signal Processing Advances in Wireless Communications, 1997.

[6] Sklar, B., Digital Communications Fundamentals and Applications, 2nd Edition, Prentice Hall, 2000.

[7] Sklar, B., Rayleigh Fading Channels in Mobile Digital Communication Systems Part I: Characterization, IEEE Communications Magazine, 1997.

[8] Jensen, F.B., W. Kuperman, M.Porter, and H. Schmidt, Computational Ocean Acoustics, American Institute of Physics, 1994.

[9] Holmes, E., Use of the Navy Standard Parabolic Equation Model for Broadband Applications, Proceedings of OCEANS '92. 'Mastering the Oceans Through Technology', 1992.

[10] Kuttler, J.R., G.D. Dockery, Theoretical Description of the Parabolic Approximation/Fourier Split-step Method of Representing Electromagnetic Propagation in the Troposphere, Radio Science, 1990. 